\title{
Theoretical prediction of hysteretic rubber friction in ball on plate configuration by finite element method
}

\author{
L. Pálfi*, T. Goda, K. Váradi \\ Department of Machine and Product Design, Budapest University of Technology and Economics, Múegyetem rkp. 3., \\ H-1111, Budapest, Hungary
}

Received 15 June 2009; accepted in revised form 26 August 2009

\begin{abstract}
This paper has investigated theoretically the influence of sliding speed and temperature on the hysteretic friction in case of a smooth, reciprocating steel ball sliding on smooth rubber plate by finite element method (FEM). Generalized Maxwell-models combined with Mooney-Rivlin model have been used to describe the material behaviour of the ethylenepropylene-diene-monomer (EPDM) rubber studied. Additionally, the effect of the technique applied at the parameter identification of the material model and the number of Maxwell elements on the coefficient of friction (COF) was also investigated. Finally, the open parameter of the Greenwood-Tabor analytical model has been determined from a fit to the FE results. By fitting, as usual, the Maxwell-model to the storage modulus master curve the predicted COF, in a broad frequency range, will be underestimated even in case of 40-term Maxwell-model. To obtain more accurate numerical prediction or to provide an upper limit for the hysteretic friction, in the interesting frequency range, the Maxwell parameters should be determined, as proposed, from a fit to the measured loss factor master curve. This conclusion can be generalized for all the FE simulations where the hysteresis plays an important role.
\end{abstract}

Keywords: rubber, FE modelling and simulation, hysteretic friction, viscoelasticity

\section{Introduction}

In the design of many mechanical engineering applications it is important to know the mechanical behaviour and tribological properties of components sliding on each other. The situation is the same if one of the sliding surfaces is made of rubber or a rubber-like material, such as sliding seals contacting with a metal or polymer surface, sliding contact between a windscreen wiper and the windscreen, or contact between a tyre and the asphalt. For the majority of sliding components applied in mechanical engineering practice, the non-lubricated operation is avoided; however, the quantity of lubricant required in maintenance free machine components is even more frequently minimized by special coatings. In the event that there is no lubricant between the rubber and the counter surface, friction is due to two physical phenomena. One of them is adhesion between the surfaces, the other one is hysteresis loss in the rubber [1-4]. For instance, if the rubber slides on a rough surface which is rigid compared to the rubber, the asperities of the counter surface repeatedly deform the surface of the rubber, leading to energy loss due to the internal friction (hysteresis) of the rubber. In this case, the energy transformed into heat as a consequence of hysteresis must be continuously supplemented if the sliding speed is intended to be constant. The majority of rubbing components made of rubber and applied in machine design operate under lubrication, to separate rubbing surfaces and thereby to reduce the impact of adhesion, as well as to reduce the exciting effect of asperities by filling up the valleys with the lubricant. When 
lubricant is used, the lubricant itself also acts as a source of friction because force is needed for shearing of the fluid film. In general, the fluid film is extremely thin, therefore in reality boundary lubrication occurs, where the source of friction is the shearing of the fluid film and of the boundary layer. In the literature, a number of articles deal with the analytic and semi-analytic determination of friction force induced by from hysteresis. Grosch [1] perform pioneering work by revealing that the two main sources of the friction force are adhesion between the surfaces and the energy loss generated in the material, that is, hysteresis. In addition, he stated that both physical phenomena are closely connected to internal friction (hysteresis) of the rubber.

Persson [2] and Klüppel and Heinrich [4] studied rubber when it is sliding on a hard, rough substrate and the surface asperities of the substrate exert oscillating forces on the rubber surface leading to energy 'dissipation' via the internal friction of the rubber. This contribution to the friction force was estimated and the results were compared with the experimental data of Grosch. Persson concluded that rubber friction on rough surfaces, in presence of lubricant, is mainly due to the viscoelastic deformations of rubber.

In spite of intensive research work in the subject, so far there have been only few attempts to predict hysteretic friction by the finite element (FE) method. For instance, in $[5,6]$ the hysteretic friction at asperity level was studied by $\mathrm{FE}$ technique. When modelling rough surfaces the topography of the 'real' surface can be replaced by sine waves or their combination. In [7] the rough surface was modelled by a single sine wave, while [8] presented a study on the combination of sine waves by using the repetitive symmetry. In the latter case, the effect of the viscoelastic material model on the predicted coefficient of friction was also investigated. In addition, [8] consists of recommendations for the parameter identification of the generalized Maxwellmodels.

In [9], Felhős et al. analysed friction force arising from hysteresis in case of a ball rolling on a rubber plate. Measurements were also modelled by FE method, where a 15-term generalized Maxwellmodel - fitted to the storage modulus master curve yielded by DMTA measurement - was used to describe viscoelastic material behaviour. His meas- urement results showed good agreement with the results of FE simulation.

In [10], the authors investigated the measurement configuration to be modelled in this study at three sliding speeds. The measurement was modelled by FE technique as well and it was investigated, using a 40-term generalized Maxwell-model fitted to the measured storage modulus master curve, what proportion of the friction force came from hysteresis. The coefficient of friction (COF) characterising lubricant shearing was determined by an iterative method and integrated into the FE model, using a prescribed Coulomb type coefficient of friction.

In measurements belonging to the current FE study (see [10]) the rubber/metal contact pair was lubricated. Consequently, the effect of adhesion on friction force can be neglected and thus rubber friction is, first of all, due to hysteresis at macroscopic and microscopic level, fluid shearing and boundary lubrication. This study is intended to estimate, using the FE technique, the hysteresis dissipation excited by an ideally smooth steel ball (macroscopic hysteretic friction) performing reciprocating sliding motion in the function of sliding speed and temperature by applying various Maxwell-models. In other words, the aim of $\mathrm{FE}$ analysis is to estimate contribution of the macroscopic hysteresis to the friction force.

\section{Description of the FE model}

The experiment is intended to determine, on the one hand, the magnitude of friction force generated by a steel ball sliding on a rubber plate, as a consequence of the internal friction of the rubber (hysteresis), at various sliding speeds and temperatures; and on the other hand, to establish how calculation results are influenced by the technique applied for parameter identification of generalized Maxwellmodels - used for the FE modelling of rubber-like materials and describing viscoelastic behaviour and by the number of the Maxwell elements. The configuration studied consists of a steel bearing ball and a rubber plate [10] where the steel ball of $2 \mathrm{~mm}$ diameter was pressed into an EPDM rubber plate of $10 \mathrm{~mm} \times 4 \mathrm{~mm} \times 2 \mathrm{~mm}$. Then the ball was forced to perform reciprocating motion with an amplitude of $A=0.3 \mathrm{~mm}$ at different, sinusoidally varying speeds through six cycles. The normal force was $F_{n}=100 \mathrm{mN}$ while the maximums of the 
sinusoidally varying sliding speeds were $v_{\max }=$ $0.01,0.1,1,10$, and $100 \mathrm{~mm} / \mathrm{s}$.

The ball, pressed into the surface of the rubber and sliding thereon, generates various COFs due to the hysteresis depending on the excited volume and on the sliding speed, that is, the excitation frequency. In order to predict the friction resistance caused by hysteresis, 3D FE modes were developed in the MSC.MARC FE system [11].

In order to describe the viscoelastic behaviour of rubber, a 15-term and a 40-term generalized Maxwell-model were used, which were produced by being fitted to storage modulus master curves determined by DMTA measurements and pertaining to $-50,+25$ and $+150^{\circ} \mathrm{C}$, respectively, and, through subsequent manual modification, by being fitted to a loss factor master curve.

The FE model was built in MSC.MARC finite element system. The rubber plate was modelled using incompressible $(v=0.5)$ elements, whereas the steel ball was modelled as an ideally rigid component. To spare with the CPU time only half of the rubber plate was modelled using symmetry condition, so the nodes in the symmetry plane were fixed in $z$-direction (Figure 1). The bottom of the rubber plate was fixed, i.e. the nodes on this plane were constrained in $x$-, $y$ - and $z$-direction.

At first, the steel ball was pressed downwards (in negative $y$-direction) into the rubber plate with the force specified, that was built up linearly. Afterwards, the ball was drawn horizontally (in negative $x$-direction) at sinusoidally varying speed according to the measurement [10], at an amplitude of $A=0.3 \mathrm{~mm}$, through six cycles. Figure 2 shows the position of the ball as a function of time in the case of a maximum sliding speed of $1 \mathrm{~mm} / \mathrm{s}$. The excitation frequency induced by the ball performing reciprocating motion can be estimated by the Equation (1):

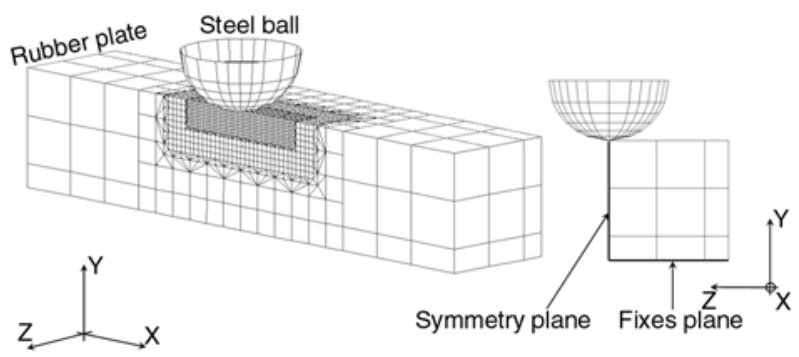

Figure 1. FE model (only half of the rubber plate was modelled due to the symmetry)

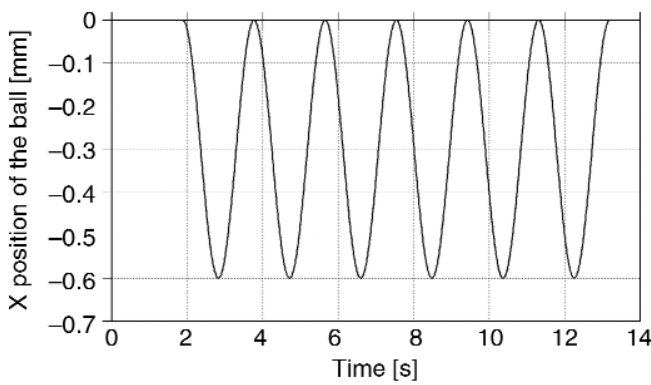

Figure 2. Displacement control curve in the direction $x$, pertaining to the maximum sliding speed of $v_{\max }=1 \mathrm{~mm} / \mathrm{s}$

$f=\frac{2}{2 \cdot \frac{\pi}{\omega}}=\frac{v_{\max }}{\pi \cdot A}$

where $\omega$ is the angular frequency, and $A$ is the amplitude of the reciprocating sliding ball. The grey band in Figures 4-9 indicates the range between the lowest and the highest frequency to be calculated from the equation above.

\subsection{Viscoelastic material model}

In order to be able to model the mechanical behaviour of structural components made of rubber or rubber-like material, first of all a material model is required which can describe both the static and dynamic behaviour of the material at the same time. The mechanical behaviour of rubber-like materials is principally characterized by a non-linear stressstrain curve and time and temperature dependency. In the present study, a large strain generalized Maxwell model (Figure 3) was used to describe the complex material behaviour of rubber. The shorttime (instantaneous) behavior of the generalized Maxwell-model applied is specified by an energy density function $\left(W^{0}\right)$ that is given, in our case, by a two-term Mooney-Rivlin material model. Thus our

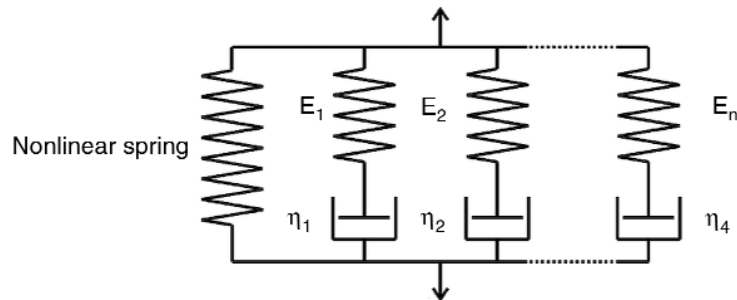

Figure 3. The n-term generalized Maxwell-model ( $E_{i}-$ elastic modulus of the $i$-th spring, $\eta_{i}-$ viscosity of the $i$-th dashpot) 
large-strain viscoelastic model shows nonlinearity between stresses and strains that, at the same time, depends on time. The instantaneous strain energy density is distributed among the branches of our spring-dashpot model by assuming that the instantaneous strain energy in the $i$-th Maxwell element can be calculated by Equation (2):

$$
W_{i}=e_{i} \cdot W^{0}
$$

where $e_{i}$ is the non-dimensional energy parameter of the $i$-th Maxwell element and $W^{0}$ is the instantaneous strain energy defined by the Mooney-Rivlin model. The interrelation among the energy parameters can be written according to Equation (3):

$$
e_{\infty}+\sum_{i=1}^{N} e_{i}=1
$$

where $e_{\infty}$ is the energy parameter of the spring that determines the relaxed response of the rubber connected parallel to the Maxwell elements and $N$ is the number of the Maxwell branches. As it is resulted from the above expressions the second Piola-Kirchhoff stress response of the generalized Maxwell model applied, in case of uniaxial tension/compression, can be expressed by Equations (4) and (5):

$$
\begin{aligned}
& S_{i}(t)=S_{\infty}(t)+\sum_{i=1}^{M} S_{i}(t) \\
& S_{i}(t)=\int_{\tau=0}^{\tau=t} e_{i} \cdot S^{0}(t) \cdot e^{-\frac{(t-\tau)}{\lambda}} \mathrm{d} \tau
\end{aligned}
$$

where $S_{\infty}(t)$ is the stress in the separate spring, $S_{i}(t)$ is the stress in the $i$-th Maxwell element or branch, $S^{0}(t)$ is the stress response of the model for instantaneous deformations and $\lambda_{i}$ is the relaxation time of the $i$-th Maxwell element. As it is known from continuum mechanics stresses can be calculated by differentiation of the proper strain energy density function with respect to the Green-Lagrange strains. For instance, $S^{0}(t)$ is obtained by differentiation of $W^{0}$. For more detail on the large strain viscoelastic model applied see [11].

The DMTA measurement results of EPDM rubber in [9] were used for producing the $n$-term Maxwellmodel.

DMTA measurements yield storage modulus vs. frequency and loss factor vs. frequency isotherms, from which a storage modulus vs. frequency and a loss factor vs. frequency master curve can be constructed at the previously selected reference temperature using the time-temperature superposition principle. These master curves show the mechanical properties of rubber in a broad frequency range at the reference temperature. The ViscoData software [12] was applied to fit 15- and 40-term generalized Maxwell-models to the storage modulus master curve produced. In order to take all the three different temperatures into account, three master curves were produced for each material model, and the material models were fitted to them separately. The reason why the authors used three different material model at the three different temperatures $\left(T=-50,25\right.$ and $150^{\circ} \mathrm{C}$ ) is that the WLF equation with universal constants $\left(C_{1}=17.4, C_{2}=51.6^{\circ} \mathrm{C}\right)$ provided inaccurate material behavior at temperatures significantly larger than the glass transition temperature $\left(T_{g} \approx-45^{\circ} \mathrm{C}\right)$. The WLF equation was able to describe the temperature dependency of the material behavior when the temperature is close to $T_{g}$. At temperatures far away $T_{g}$ (eg. at $T=25^{\circ} \mathrm{C}$ ) WLF overestimated considerably the horizontal shift needed. Instead of making attempt to find proper $C_{1}, C_{2}$ constants for the WLF equation three different master curves were created at the three different temperatures studied. Figure 4 and Figure 5 show the measured and fitted storage modulus vs. frequency curves, while Figure 6 and Figure 7 show the loss factor vs. frequency curves in case of the 15- and 40-term generalized Maxwellmodels fitted to the storage modulus, at three different temperatures.

The magnitude of hysteretic friction is basically determined by two factors: one of them is the magnitude of the loss factor, and the other is the excited volume. Hysteretic dissipation is determined by the joint effect of these two factors. The first factor can obviously be handled by a good correspondence between the measured loss factor vs. frequency curve and the one used in modelling; while the second factor is determined by the storage modulus, that is, how rigid the material is and how deeply the rigid counter surface can penetrate into it, meaning the volume forced to deform.

It is intended to produce a viscoelastic model which closely approaches both the storage modulus vs. frequency and the loss factor vs. frequency curves measured. 


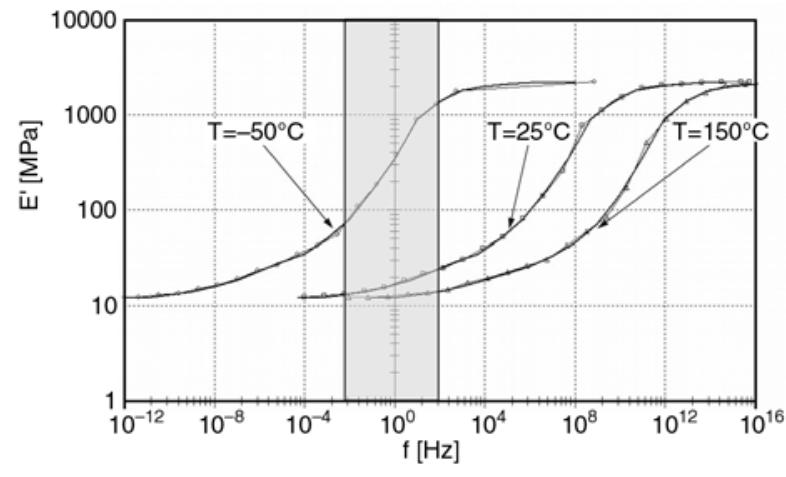

Figure 4. Comparison of the measured and simulated storage modulus vs. frequency curves in case of a 15-term generalized Maxwell-model fitted to the storage modulus ( strain amplitude $=0.01 \%$ ).

- measurement $T=-50^{\circ} \mathrm{C}, \vartheta T=-50^{\circ} \mathrm{C}$, fitted to the $E^{\prime}$, - measurement $T=25^{\circ} \mathrm{C}$,

$\square T=25^{\circ} \mathrm{C}$, fitted to the $E^{\prime}$, - measurement $T=150^{\circ} \mathrm{C}, \triangle T=150^{\circ} \mathrm{C}$, fitted to the $E^{\prime}$.

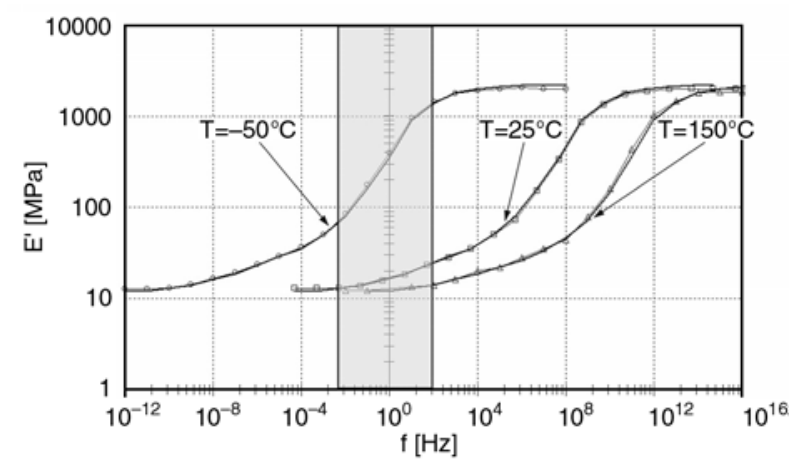

Figure 5. Comparison of the measured and simulated storage modulus vs. frequency curves in case of a 40-term generalized Maxwell-model fitted to the storage modulus (strain amplitude $=0.01 \%$ ).

- measurement $T=-50^{\circ} \mathrm{C}, \vartheta T=-50^{\circ} \mathrm{C}$, fitted to the $E^{\prime}$, - measurement $T=25^{\circ} \mathrm{C}$,

$\square T=25^{\circ} \mathrm{C}$, fitted to the $E^{\prime},-$ measurement $T=150^{\circ} \mathrm{C}, \triangle T=150^{\circ} \mathrm{C}$, fitted to the $E^{\prime}$.

In case of the 15-term generalized Maxwell-model it can be observed that there is good correspondence between the measurement and the fitted models as regards the storage modulus (Figure 4), but the loss factor produces large oscillation (Figure 6). By increasing the number of Maxwell elements to 40, the fluctuation becomes smaller; however, even in this case, the model strongly underestimates the value of the loss factor within a broad frequency range (Figure 7). It can be stated that the 15-term generalized Maxwell-model cannot describe, with adequate accuracy, either the nature of the loss factor or its numerical value. The generalized Maxwell-model fitted to a 40-term storage modulus already properly represents the

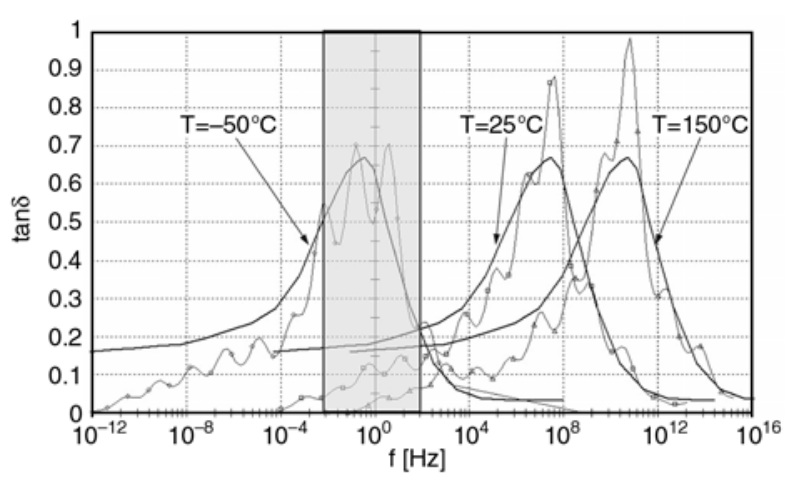

Figure 6. Comparison of the measured and simulated loss factor vs. frequency curves in case of a 15-term generalized Maxwell-model fitted to the storage modulus.

- measurement $T=-50^{\circ} \mathrm{C}, \vartheta T=-50^{\circ} \mathrm{C}$, fitted to the $\tan \delta,-$ measurement $T=25^{\circ} \mathrm{C}$,

$\square T=25^{\circ} \mathrm{C}$, fitted to the tan $\delta,-$ measurement $T=150^{\circ} \mathrm{C}, \triangle T=150^{\circ} \mathrm{C}$, fitted to the tan $\delta$.

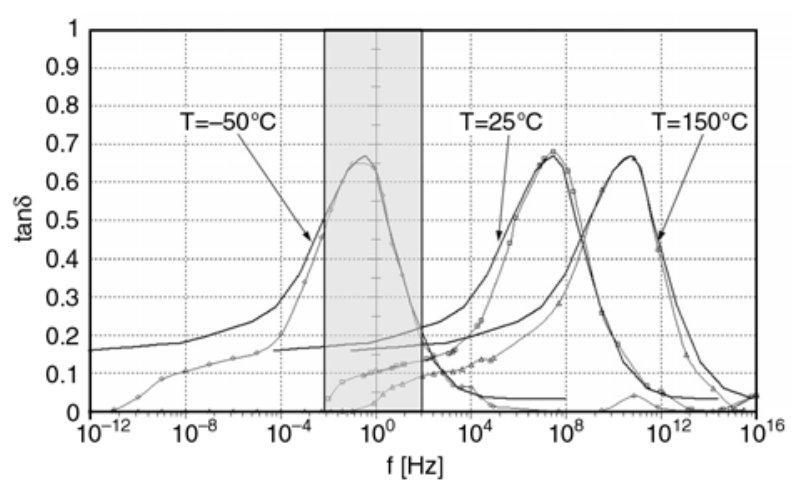

Figure 7. Comparison of the measured and simulated loss factor vs. frequency curves in case of a 40 -term generalized Maxwell-model fitted to the storage modulus.

- measurement $T=-50^{\circ} \mathrm{C}, \vartheta T=-50^{\circ} \mathrm{C}$, fitted to the $\tan \delta,-$ measurement $T=25^{\circ} \mathrm{C}$,

$\square T=25^{\circ} \mathrm{C}$, fitted to the $\tan \delta$, - measurement $T=150^{\circ} \mathrm{C}, \triangle T=150^{\circ} \mathrm{C}$, fitted to the tan $\delta$.

loss factor vs. frequency curve determined from the measurement in terms of quality - and also in terms of quantity at most frequencies - but it still cannot provide an adequate numerical solution in case of certain frequencies. It can be further stated that following adjustment of the material model it is not sufficient to be satisfied with a good correspondence between the storage modulus curves of the measured and fitted material models, but it is necessary to check the loss factor vs. frequency curve as well. No reliable hysteretic dissipation can be calculated by the FE technique unless the material model can properly represent both the storage modulus and loss factor master curves determined by measurement. In order to model the measured 


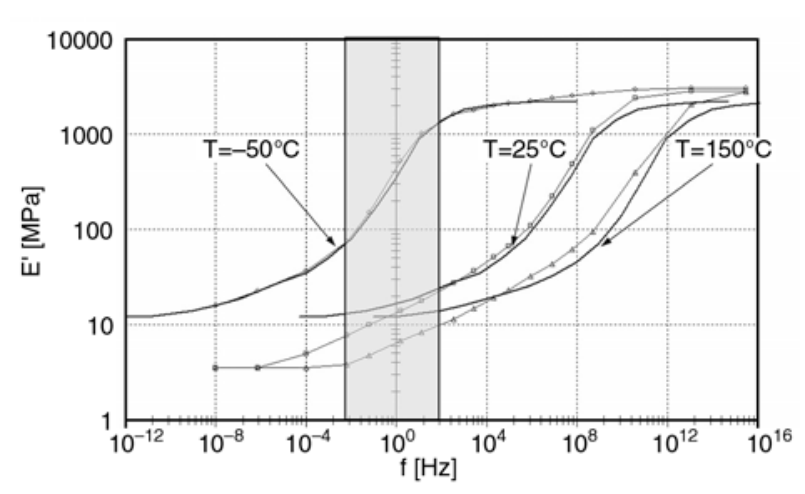

Figure 8. Comparison of the measured and simulated storage modulus vs. frequency curves in case of a 40-term generalized Maxwell-model fitted to the loss factor ( strain amplitude $=0.01 \%$ ).

- measurement $T=-50^{\circ} \mathrm{C}, \vartheta T=-50^{\circ} \mathrm{C}$, fitted to the $E^{\prime}$, - measurement $T=25^{\circ} \mathrm{C}$, $T=25^{\circ} \mathrm{C}$, fitted to the $E^{\prime},-$ measurement $T=150^{\circ} \mathrm{C}, \triangle T=150^{\circ} \mathrm{C}$, fitted to the $E^{\prime}$.

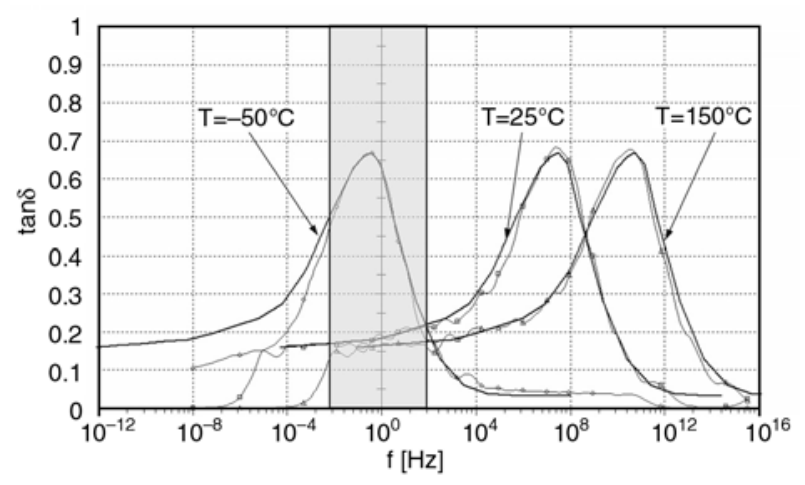

Figure 9. Comparison of the measured and simulated loss factor vs. frequency curves in case of a 40-term generalized Maxwell-model fitted to the loss factor.

- measurement $T=-50^{\circ} \mathrm{C}, \vartheta T=-50^{\circ} \mathrm{C}$, fitted to the $\tan \delta,-$ measurement $T=25^{\circ} \mathrm{C}$, $\square T=25^{\circ} \mathrm{C}$, fitted to the tan $\delta,-$ measurement $T=150^{\circ} \mathrm{C}, \triangle T=150^{\circ} \mathrm{C}$, fitted to the tan $\delta$.

material behaviour more accurately, a 40-term Maxwell-model was produced by manual modification [8], fitted to the loss factor. Figure 8 and Figure 9 show, at three different temperatures, the measured storage modulus vs. frequency and loss factor vs. frequency curves of the 40-term generalized Maxwell-model and those fitted to the loss factor.

In the course of adjustment to the loss factor, correspondence with the storage modulus curve will not be as good as in the case of adjusting the material model to the measured storage modulus master curve. The parameters of the material model can be considered as optimal when the model can describe, with acceptable accuracy, both master curves $\left(E^{\prime}\right.$, $\tan \delta$ ) determined by measurement. Figure 9 shows that as a result of manual modification, the material model can describe the measured $\tan \delta$ curve with acceptable accuracy. Furthermore, it can be stated in the case of all the three Maxwell-models that the material models cannot describe the storage modulus and the loss factor master curves with constant accuracy within the entire frequency range, therefore care must be taken at what excitation frequency and at what temperature the material model is used.

\subsection{Non-linear material model}

In the FE model, the non-linear stress-strain curve of rubber was defined by the frequently applied Mooney-Rivlin material law with two parameters. The two Mooney-Rivlin parameters were defined on the basis of [11], using the Equations (6) and (7):

$$
\begin{aligned}
& E_{0}=6 \cdot\left(C_{01}+C_{10}\right) \\
& \frac{C_{01}}{C_{10}}=\frac{1}{4}
\end{aligned}
$$

where $E_{0}$ is the glassy modulus. The two parameters used for the calculations were $C_{10}=$ $289.33 \mathrm{MPa}$ and $C_{01}=72.33 \mathrm{MPa}$ in case of the 15- and 40-term generalized Maxwell-models fitted to the storage modulus; their value was $C_{10}=$ 406.66 $\mathrm{MPa}$ and $C_{01}=101.66 \mathrm{MPa}$, respectively, in case of the generalized Maxwell-model fitted to the loss factor. The non-dimensional energy parameters $\left(e_{i}\right)$ of the Maxwell model fitted to the loss factor $(\tan \delta)$ master curve were obtained by manual modification from the energy parameters of the Maxwell model fitted to the storage modulus master curve. Due to this modification the sum of the energy parameters of the Maxwell elements ( $\sum_{i=1}^{40} e_{i}$ ) has been changed. Consequently, if we used the same glassy Mooney parameters in both cases, we would obtain an accurate relaxed modulus at the Maxwell model fitted to $E^{\prime}$ while, at the Maxwell model fitted to $\tan \delta$, the relaxed modulus would be considerably underestimated in the interesting frequency range. To minimize this underestimation i.e. to approach $E^{\prime}$ the real values, the glassy Mooney parameters have been changed. Thus we obtain higher glassy modulus than the measured one but the agreement, within the interesting fre- 
quency range, between the modeled and measured $E^{\prime}$ values will be better than in the case of same Mooney parameters. In our case, the change in $\sum_{i=1}^{40} e_{i}$ at $-50^{\circ} \mathrm{C}$ was smaller than at 25 and $150^{\circ} \mathrm{C}$ as it can be seen in Figure 8 . At $T=25^{\circ} \mathrm{C}$ (see Figure 7) the Maxwell model fitted to the $E^{\prime}$ master curve by ViscoData, contrary to the measurement, provides zero or very small loss factor values at low frequencies. As it can be seen in Figure 9 the new model (fitted to $\tan \delta$ ) created by the manual modification of Maxwell parameters provided by ViscoData shows good agreement with the measured $\tan \delta$ curve. During creation of the new material model authors had to redistribute one part of the relaxation times also.

\section{Results and discussion}

\subsection{FE results}

Tables 1-3 show the penetration depths $(h)$ and the radius of the contact area (a) calculated by the FE technique and the COFs induced by hysteresis $\left(\mu_{\text {hysteresis }}\right)$. In case of the 15-term Maxwell-model it can be observed that at $-50^{\circ} \mathrm{C}$, the penetration depth of the ball reduces from 9.7 to $1.78 \mu \mathrm{m}$ as the speed increases, due to the fact that the storage modulus of the material rises as the speed increases. For similar reasons, a decrease of penetration depth by the increase of speed can be observed in case of all the material models and temperatures applied. In case of the 15- and 40-term material models fitted to the storage modulus, the value of the penetration depth is approximately the same, but in case of the 40-term material model fitted to the loss factor, penetration depth may be as much as $60 \%$ bigger at low speeds as a result of manual modification. Taking speeds into consideration, it can bee stated that the biggest penetration values can be calculated at $150^{\circ} \mathrm{C}$. However, as it can be seen in Table 1 and 2, at low speeds $(v=0.01$ and $0.1 \mathrm{~mm} / \mathrm{s})$ differences between the penetration values calculated at $T=25$ and $150^{\circ} \mathrm{C}$ are within accuracy of the numerical solution.

As regards COFs resulting from hysteresis, it can be stated that in case of material models fitted to the storage modulus the FE model surely underestimates hysteresis as there is a considerable difference between the loss factor curves measured and those defined by the material model. Exceptions only include the COFs provided at $-50^{\circ} \mathrm{C}$ by the 40-term Maxwell-model adjusted to $E^{\prime}$ (see Table 2). In this case, there is good correspondence between the measured and the simulated material behaviour both as regards $E^{\prime}$ and $\tan \delta$ (Figure 5 and

Table 1. Values of the penetration depth $(h)$, radius of the contact area $(a)$ and the hysteretic coefficient of friction ( $\mu_{\text {hysteresis }}$ ) in case of 15-term Maxwell-model fitted to the measured storage modulus master curve

\begin{tabular}{|c|c|c|c|c|c|c|}
\hline Temperature $\left[{ }^{\circ} \mathbf{C}\right]$ & $\mathbf{v}_{\text {max }}[\mathbf{m m} / \mathbf{s}]$ & $\mathbf{0 . 0 1}$ & $\mathbf{0 . 1}$ & $\mathbf{1}$ & $\mathbf{1 0}$ & $\mathbf{1 0 0}$ \\
\hline \multirow{3}{*}{50} & $h[\mu \mathrm{m}]$ & 9.70 & 6.67 & 4.13 & 2.47 & 1.78 \\
\cline { 2 - 7 } & $a[\mu \mathrm{m}]$ & 98.47 & 81.68 & 64.28 & 49.65 & 42.19 \\
\cline { 2 - 7 } & $\mu_{\text {hysteresis }}$ & 0.0180 & 0.0160 & 0,0090 & 0,0050 & 0.0040 \\
\hline \multirow{3}{*}{25} & $h[\mu \mathrm{m}]$ & 27.03 & 25.94 & 24.12 & 23.06 & 19.93 \\
\cline { 2 - 7 } & $a[\mu \mathrm{m}]$ & 164.40 & 161.07 & 155.29 & 151.86 & 141.18 \\
\cline { 2 - 7 } & $\mu_{\text {hysteresis }}$ & 0.0051 & 0.0073 & 0.0108 & 0.0118 & 0.0113 \\
\hline \multirow{3}{*}{150} & $h[\mu \mathrm{m}]$ & 26.16 & 26.16 & 26.04 & 25.61 & 24.87 \\
\cline { 2 - 7 } & $a[\mu \mathrm{m}]$ & 161.75 & 161.74 & 161.37 & 160.04 & 157.69 \\
\cline { 2 - 7 } & $\mu_{\text {hysteresis }}$ & 0.0001 & 0.0006 & 0.0029 & 0.0041 & 0.0073 \\
\hline
\end{tabular}

Table 2. Values of the penetration depth $(h)$, radius of the contact area $(a)$ and the hysteretic coefficient of friction ( $\mu_{\text {hysteresis }}$ ) in case of 40-term Maxwell-model fitted to the measured storage modulus master curve

\begin{tabular}{|c|c|c|c|c|c|c|}
\hline Temperature $\left[{ }^{\circ} \mathbf{C}\right]$ & $\mathbf{V}_{\text {max }}[\mathbf{m m} / \mathbf{s}]$ & $\mathbf{0 . 0 1}$ & $\mathbf{0 . 1}$ & $\mathbf{1}$ & $\mathbf{1 0}$ & $\mathbf{1 0 0}$ \\
\hline \multirow{3}{*}{50} & $h[\mu \mathrm{m}]$ & 6.70 & 5.38 & 5.11 & 3.55 & 4.20 \\
\cline { 2 - 7 } & $a[\mu \mathrm{m}]$ & 83.65 & 73.32 & 71.45 & 59.60 & 64.71 \\
\cline { 2 - 7 } & $\mu_{\text {hysteresis }}$ & 0.0120 & 0.0080 & 0.0050 & 0.0020 & 0.0007 \\
\hline \multirow{3}{*}{25} & $h[\mu \mathrm{m}]$ & 25.94 & 25.60 & 24.59 & 22.78 & 20.65 \\
\cline { 2 - 7 } & $a[\mu \mathrm{m}]$ & 161.04 & 159.85 & 156.82 & 150.93 & 143.71 \\
\cline { 2 - 7 } & $\mu_{\text {hysteresis }}$ & 0.0041 & 0.0078 & 0.0087 & 0.0106 & 0.0111 \\
\hline \multirow{3}{*}{150} & $h[\mu \mathrm{m}]$ & 25.90 & 25.90 & 25.88 & 25.56 & 24.74 \\
\cline { 2 - 7 } & $a[\mu \mathrm{m}]$ & 160.94 & 160.94 & 160.89 & 159.86 & 157.30 \\
\cline { 2 - 7 } & $\mu_{\text {hysteresis }}$ & 0.0004 & 0.0010 & 0.0031 & 0.0079 & 0.0088 \\
\hline
\end{tabular}


Table 3. Values of the penetration depth $(h)$, radius of the contact area $(a)$ and the hysteretic coefficient of friction $\left(\mu_{\text {hysteresis }}\right)$ in case of 40-term Maxwell-model fitted to the measured loss factor master curve

\begin{tabular}{|c|c|c|c|c|c|c|}
\hline Temperature $\left[{ }^{\circ} \mathbf{C}\right]$ & $\mathbf{v}_{\text {max }}[\mathbf{m m} / \mathbf{s}]$ & $\mathbf{0 . 0 1}$ & $\mathbf{0 . 1}$ & $\mathbf{1}$ & $\mathbf{1 0}$ & $\mathbf{1 0 0}$ \\
\hline \multirow{3}{*}{50} & $h[\mu \mathrm{m}]$ & 9.74 & 6.62 & 4.02 & 2.54 & 1.82 \\
\cline { 2 - 7 } & $a[\mu \mathrm{m}]$ & 98.68 & 81.35 & 63.38 & 50.40 & 42.72 \\
\cline { 2 - 7 } & $\mu_{\text {hysteresis }}$ & 0.0210 & 0.0180 & 0.0070 & 0.0030 & 0.0008 \\
\hline \multirow{3}{*}{25} & $h[\mu \mathrm{m}]$ & 42.51 & 36.28 & 30.79 & 25.79 & 21.61 \\
\cline { 2 - 7 } & $a[\mu \mathrm{m}]$ & 206.18 & 190.48 & 175.48 & 160.60 & 147.02 \\
\cline { 2 - 7 } & $\mu_{\text {hysteresis }}$ & 0.0203 & 0.0177 & 0.0184 & 0.0176 & 0.0173 \\
\hline \multirow{3}{*}{150} & $h[\mu \mathrm{m}]$ & 57.22 & 55.37 & 49.44 & 42.51 & 36.28 \\
\cline { 2 - 7 } & $a[\mu \mathrm{m}]$ & 239.22 & 235.32 & 222.34 & 206.18 & 190.48 \\
\cline { 2 - 7 } & $\mu_{\text {hysteresis }}$ & 0.0208 & 0.0237 & 0.0279 & 0.0212 & 0.0177 \\
\hline
\end{tabular}

7). The calculated COFs can be considered as acceptably accurate. In this case, any subsequent modification of the Maxwell-parameters will impair the accuracy of numerical results (see the results at $-50^{\circ} \mathrm{C}$ in Table 3 ). In this case, less accurate results are yielded as a consequence of the less accurate modelling of the measured storage modulus. In case of the 15-term material model, the highest hysteresis can be observed at $-50^{\circ} \mathrm{C}$ at the first two speeds, in spite of a small penetration depth, as a result of a high loss factor. At 25 and $150^{\circ} \mathrm{C}$, hysteresis increases as the speed increases, as suggested by the rising loss factor curves (Figure 6). In case of the 40-term Maxwell-model fitted to the storage modulus, the highest hysteresis can be calculated at $-50^{\circ} \mathrm{C}$ at the lowest speed (Table 2), due to the high loss factor. In case of the other speeds, hysteresis is the highest at $25^{\circ} \mathrm{C}$, increasing as the speed increases. In case of the 40-term Maxwellmodel fitted to the loss factor (see Table 3 ) the highest hysteresis can be observed at $150^{\circ} \mathrm{C}$ at each speed, due to a low elastic modulus and a significant loss factor. The absolutely highest hysteresis was calculated at $1 \mathrm{~mm} / \mathrm{s}$. As shown in Figure 7 , the Maxwell-model fitted to the storage modulus can only model developments in the measured loss factor very inaccurately within the frequency range indicated by grey colour. Accordingly, the results figuring in the second and third lines of Table 2 can only be considered as rough lower estimates. To the contrary, the 40-term Maxwell-model fitted to the loss factor and shown in Figure 8 and 9 models the loss factor accurately but underestimates the measured storage modulus. In case of increasing frequencies, the degree of underestimation gradually decreases. As a result of the underestimated elastic modulus, the results in Table 3 for 25 and $150^{\circ} \mathrm{C}$ can be considered as upper estimates as regards the
COF. Based on Figure 8 and 9, it can be stated that the COF result in Table 3, pertaining to $25^{\circ} \mathrm{C}$ and $v_{\max }=100 \mathrm{~mm} / \mathrm{s}$ can be considered as acceptably accurate. In this case, the COF value is more than $50 \%$ higher than the COF value in the last column of the second row in Table 2, determined using the 40-term Maxwell-model fitted to the storage modulus.

\subsection{Comparison of the FE results to a simple analytical model}

The correlation to estimate the hysteretic coefficient of friction presented in [13] was used for interpreting FE results; this way changes in the coefficient of friction can be analyzed in quantitative terms. Greenwood and Tabor demonstrated that in case of a rigid ball sliding on rubber, the hysteretic coefficient of friction can be estimated by Equations (8) and (9):

$$
\begin{aligned}
& \mu=\frac{9 \cdot \pi}{64} \cdot \frac{1-v^{2}}{\hat{E}} \cdot p_{a v} \cdot \alpha \\
& \frac{1}{\hat{E}}=\frac{1-v_{1}^{2}}{E_{1}}+\frac{1-v_{2}^{2}}{E_{2}}
\end{aligned}
$$

where $v$ is the Poisson ratio; $\hat{E}$ is the equivalent elastic modulus of the contacting bodies defined by the Equation (9); $p_{a v}$ is the average contact pressure; and $\alpha$ is the number characterizing the energy dissipation resulting from hysteresis in the rubber, specifying what proportion of the work required to deform the rubber in front of the sliding ball is dissipated as a consequence of hysteresis. The authors did not publish further correlations to determine the value of $\alpha$. The average contact pressure can be calculated by Equation (10): 


$$
p_{a \vee}=\frac{F_{n}}{a^{2} \cdot \pi}
$$

where $F_{n}$ is the force on the ball, and $a$ is the radius of the contact area. To determine $\hat{E}$, the complex Young modulus was determined from the storage modulus and loss factor master curves for each given frequency according to Equation (11) and it was substituted for $\left(1-v^{2}\right) / E$ :

$$
E^{*}=\frac{E^{\prime}}{\cos \delta}
$$

If the left side of Equation (8) is replaced by the coefficients of friction calculated by the FE method ( $\left.\mu_{\text {hysteresis }}\right)$, then parameter $\alpha$ in the GreenwoodTabor model can be determined in the function of sliding speed and temperature. Figures 10 and 11
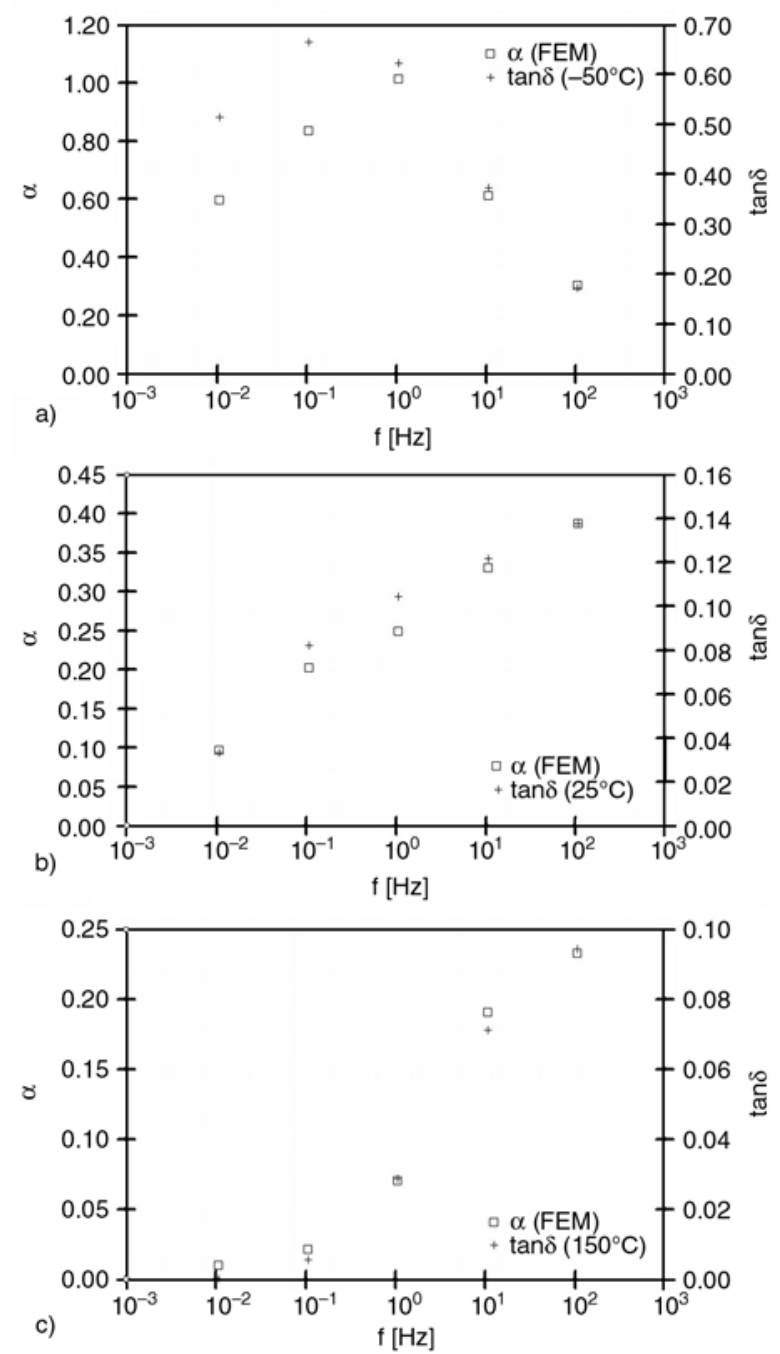

Figure 10. Variation of $\alpha$ and $\tan \delta$ in function of frequency (40-term Maxwell-model fitted to the storage modulus master curve): a) $T=-50^{\circ} \mathrm{C}$, b) $T=25^{\circ} \mathrm{C}$, c) $T=150^{\circ} \mathrm{C}$ show the frequency dependence of factor $\alpha$ and of the loss factor of the rubber with reference to 40-term material models and various temperatures. It can be observed in terms of tendency that the values of $\alpha$ properly follow the changes in $\tan \delta$ at each temperature, in the function of frequency. Greenwood and Tabor [13] made an attempt to determine $\alpha$ from cyclic tests having strain rate comparable to those occurring in sliding experiments. In their interpretation, the energy loss due to hysteresis was defined as $\alpha$ times the total elastic energy of deformation. We assumed that $\alpha$ is influenced by the phase shift between stress and strain as, in case of cyclic tension/compression, the hysteretic loss per cycle and per unit volume is influenced by $\sin \delta$. Since the tendency of $\sin \delta$ and $\tan \delta$, within the loss factor range measured by DMTA, is similar we
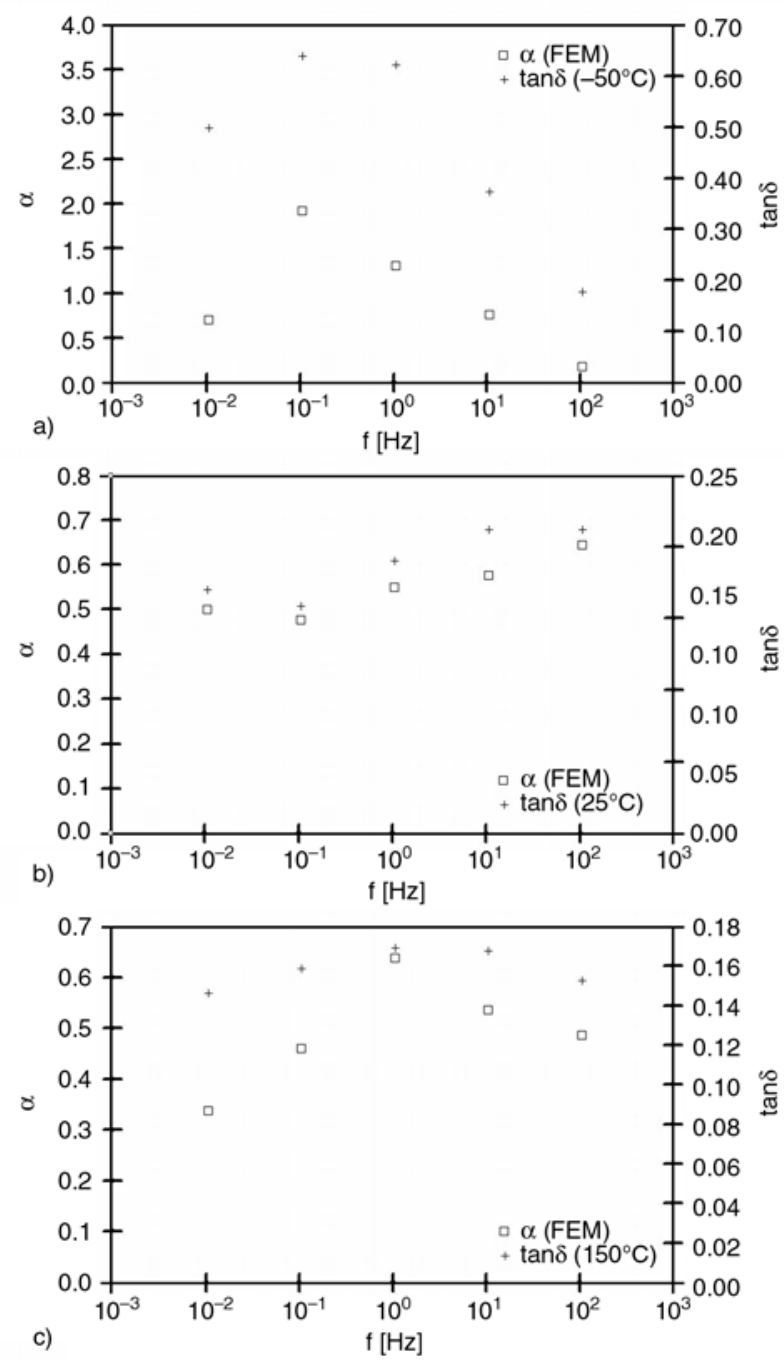

Figure 11. Variation of $\alpha$ and $\tan \delta$ in function of frequency (40-term Maxwell-model fitted to the loss factor master curve): a) $T=-50^{\circ} \mathrm{C}$, b) $T=25^{\circ} \mathrm{C}$, c) $T=150^{\circ} \mathrm{C}$ 
used $\tan \delta$ for comparison with $\alpha$. Based on these, Equation (8) can be used for a qualitative description of changes in the hysteretic coefficient of friction if $\alpha$ is substituted by $\tan \delta$.

$\mu \approx \frac{9 \cdot \pi}{64} \cdot \frac{1-\mu^{2}}{\hat{E}} \cdot p_{a v} \cdot \tan \delta$

Figures 12 and 13 compare the coefficients of friction calculated by FE method and by Equation (12). As expected, COFs calculated by Equation (12), in every case are smaller than the ones predicted by the numerical method. In respect of the tendencies, the values calculated by the two different methods are in a good agreement.
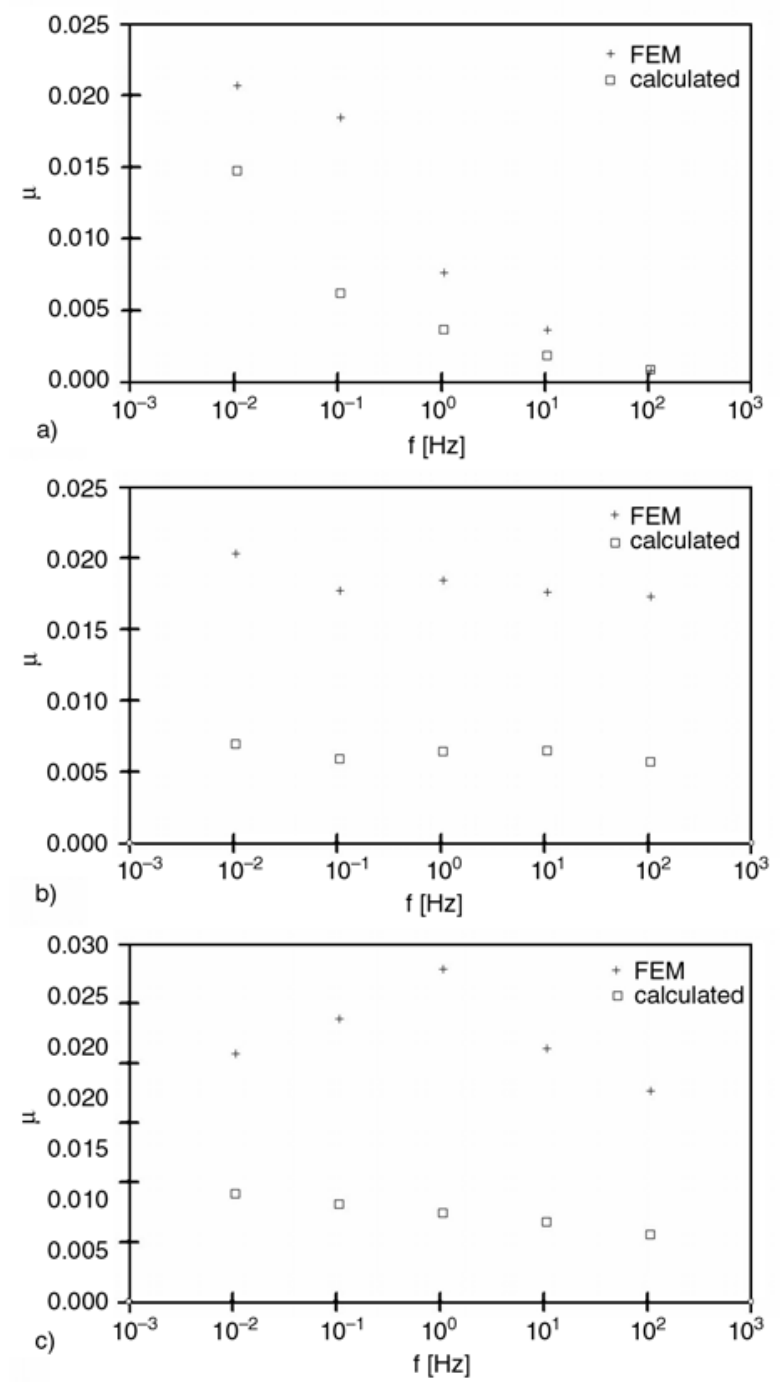

Figure 12. Comparison of the coefficients of friction predicted by FEM and Equation (8) (40-term Maxwell-model fitted to the storage modulus master curve): a) $T=-50^{\circ} \mathrm{C}$, b) $T=25^{\circ} \mathrm{C}$, c) $T=150^{\circ} \mathrm{C}$

\section{Conclusions}

1. An FE model has been developed for the prediction of hysteretic friction force in case of a reciprocating steel ball sliding on a rubber plate.

2. It can be concluded that because of the high oscillation in the tan $\delta$ curve the 15 -term generalized Maxwell-model fitted to the whole frequency range is not able to characterize the timeand temperature-dependent behaviour of the rubber. To avoid this oscillation Maxwell-models with 40-term were constructed.

3. It can also be concluded that, within a certain frequency range, even the 40-term Maxwell-model fitted to the storage modulus master curve under-
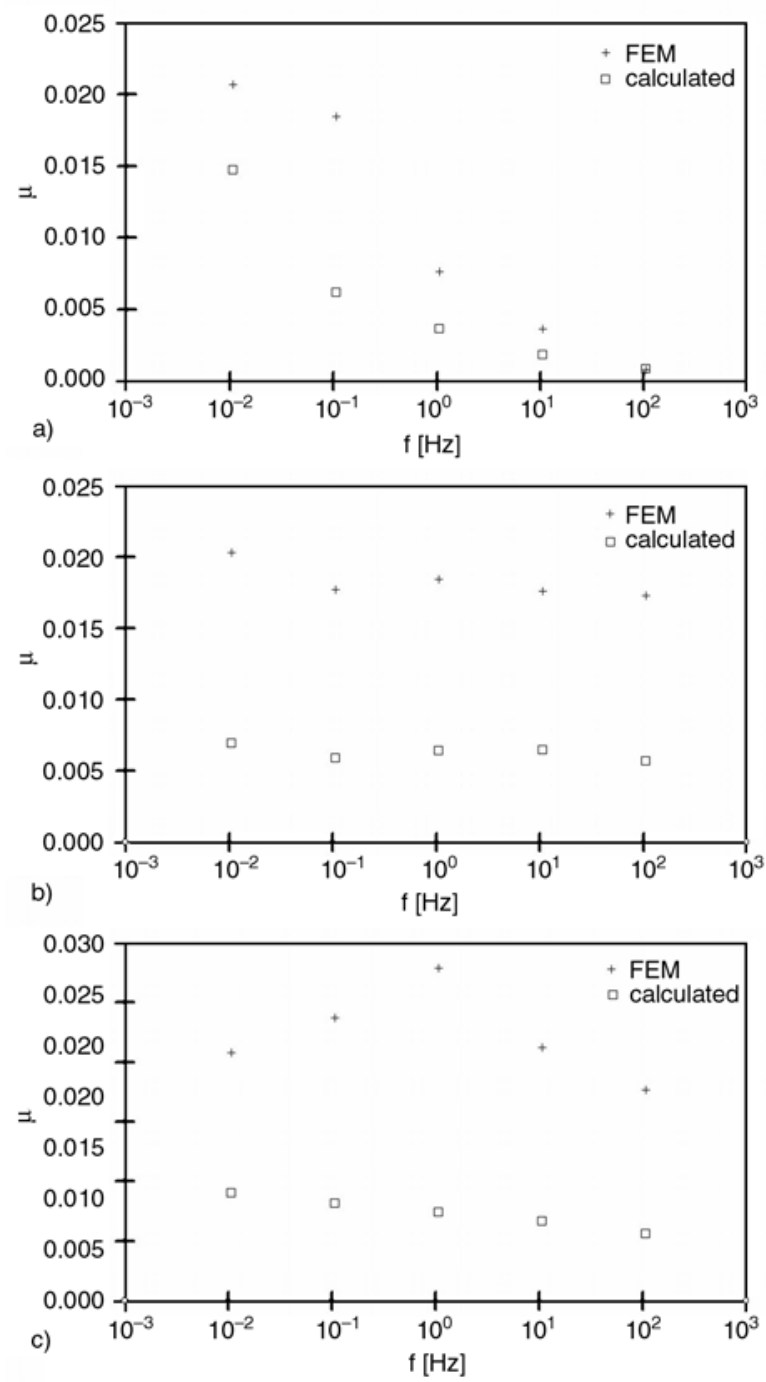

Figure 13. Comparison of the coefficients of friction predicted by FEM and Equation (8) (40-term Maxwell-model fitted to the loss factor master curve): a) $T=-50^{\circ} \mathrm{C}$, b) $T=25^{\circ} \mathrm{C}$, c) $T=150^{\circ} \mathrm{C}$ 
estimates the hysteretic friction because the loss factor provided by the material model is about the half of the one measured. The accuracy of the numerical prediction depends not only on the excitation frequency but also on the operational temperature as it shifts the master curves horizontally according to the time-temperature superposition. This conclusion is extremely important because the viscoelastic material model parameters usually are determined from a fit to the storage modulus master curve in the literature.

4. In order to predict the hysteretic friction more accurately or to provide below and/or upper limit for the hysteretic coefficient of friction one has to construct a material model that describes both the storage modulus and the loss factor master curves measured with sufficient accuracy. By using the proposed technique i.e. fitting the material model to the measured loss factor master curve the accuracy of the numerical prediction can be improved significantly in several cases.

5. From fit to the numerical predictions the open parameter of the Greenwood-Tabor model $(\alpha)$ has been determined as a function of frequency and temperature. Its value ranges from 0.01 to 2 . Without specifying parameter $\alpha$ properly the Greenwood-Tabor model can not be used to predict hysteretic friction generated by a sliding ball.

\section{Acknowledgements}

The authors wish to acknowledge the support of the Partners of the Kristal project and the European Commission for their support in the integrated project 'Knowledgebased Radical Innovation Surfacing for Tribology and Advanced Lubrication' (EU Project Reference NMP3-CT2005-515837).

\section{References}

[1] Grosch K. A.: The relation between the friction and viscoelastic properties of rubber. Proceedings of the Royal Society of London, Series A, 274, 21-39 (1963).

DOI: $10.1098 /$ rspa.1963.0112

[2] Persson B. N. J.: Theory of rubber friction and contact mechanics. The Journal of Chemical Physics, 115, 3840-3861 (2001). DOI: $\underline{10.1063 / 1.1388626}$

[3] Persson B. N. J., Albohr O., Creton C., Peveri V.: Contact area between a viscoelastic solid and a hard, randomly rough, substrate. The Journal of Chemical Physics, 120, 8779-8793 (2004).

DOI: $\underline{10.1063 / 1.1697376}$

[4] Klüppel M., Heinrich G.: Rubber friction on selfaffine road tracks. Rubber Chemistry and Technology, 73, 779-797 (2000).

[5] Bui Q. V., Ponthot J. P.: Estimation of rubber sliding friction from asperity interaction modelling. Wear, 252, 150-60 (2002).

DOI: $\underline{10.1016 / \mathrm{S} 0043-1648(01) 00864-\mathrm{X}}$

[6] Soós E., Goda T.: Numerical analysis of sliding friction behaviour of rubber. Materials Science Forum, 537-538, 615-621 (2007).

DOI: 10.4028/www.scientific.net/MSF.537-538.615

[7] Nettingsmeier J., Wriggers P.: Frictional contact of elastomer materials on rough rigid surface. PAMM, 4, 360-361 (2004). DOI: $10.1002 / \mathrm{pamm} .200410161$

[8] Pálfi L., Goda T., Váradi K., Garbayo E., Bielsa J. M., Jiménez M. A.: FE prediction of hysteretic component of rubber friction. Meccanica, in press (2009).

[9] Felhős D., Xu D., Schlarb A. K., Váradi K., Goda T.: Viscoelastic characterization of an EPDM rubber and finite element simulation of its dry rolling friction. Express Polymer Letters, 2, 157-164 (2008). DOI: 10.3144/expresspolymlett.2008.21

[10] Pálfi L., Fernández B., Váradi K.: FE modelling of oscillating sliding friction between a steel ball and an EPDM plate. in 'Proceedings of Sixth Conference on Mechanical Engineering. Budapest, Hungary' on CD p. 11 (2008).

[11] MSC. Marc user manual, Version 2007R1: MSC. Software corporation, Santa Ana (2007).

[12] Herdy M.: Introductory theory manual ViscoData and ViscoShift. IBH-Ingenierbüro, Grafschaft (2003).

[13] Greenwood J. A., Tabor D.: The friction of hard sliders on lubricated rubber: The importance of deformation losses. Proceedings of the Physical Society, 71, 989-1001 (1958).

DOI: $\underline{10.1088 / 0370-1328 / 71 / 6 / 312}$ 